\title{
Performance Analysis of a Simple Subspace Projection-based Channel Estimate Improver for Multicarrier CDMA Systems
}

\author{
Deolinda Fontes Cardoso, Fabian David Backx, and Raimundo Sampaio-Neto
}

\begin{abstract}
This paper addresses pilot-assisted estimation of frequency-selective time-invariant channels in multicarrier Code Division Multiple Access communications systems (MC CDMA and MC DS-CDMA). Performance in terms of normalised mean square error (NMSE) is derived for two discrete channel frequency response estimators: a conventional estimator based on the minimum mean square error criterion, and an improved estimator exploiting subspace relationships between the frequency and impulse responses of the discrete channel. For MC DSCDMA, NMSE performances of both estimators result in closedform solutions. For MC CDMA, a closed-form solution is derived for the NMSE of the conventional estimator; upper and lower bounds are provided for the NMSE of the improved estimator Furthermore, for the particular case of identically distributed discrete channels with common arbitrary power delay profile and uncorrelated weights, a closed-form expression for the NMSE of the improved estimator is also obtained. Numerical results illustrate the accuracy of the proposed NMSE expressions.
\end{abstract}

Index Terms-Pilot-aided Channel Estimation, Projection Matrix, MC CDMA, MC DS-CDMA.

\section{INTRODUCTION}

The fantastic growth of wireless services and multiuser communications and the increasing demand for higher data rates during the last decade have boosted the research on broadband communications systems. The Direct Sequence Code Division Multiple Access (DS-CDMA) technique has already been successfully implemented for third generation mobile communication systems, mainly due to its ability to efficiently share the bandwidth amongst several simultaneously transmitting narrowband users without (substantial) deterioration on either systems performance [1], [2], [3], [4]. However, as data rates increase, the communications channels may become very hostile and induce Inter-Symbol Interference. Also, multiple access interference (MAI) which stems from the lack of code orthogonality at reception and the so-called near-far effect which find it sources in imperfect power control and MAI itself, hinder higher effective data throughput.

Orthogonal Frequency Division Multiplexing (OFDM) is an ingenious mature technique [5], [6], [7], [8] which overcomes channel delay spreads and achieves higher spectrum efficiency in wireless communications systems. OFDM entails redundant

D. F. Cardoso is with Centro de Análises de Sistemas Navais, Brazil (email: deolinda@cetuc.puc-rio.br).

F. D. Backx is with Instituto de Pesquisas da Marinha, Brazil (email: fdb@cetuc.puc-rio.br).

R. Sampaio-Neto is with Centro de Estudos em Telecomunicações da Pontifícia Universidade Católica do Rio de Janeiro, Brazil (email: raimundo@cetuc.puc-rio.br) block transmissions and enables very simple equalisation of frequency-selective finite impulse response (FIR) channels using single-tap equalisers. A frequency-selective channel is converted into parallel independent subchannels, each experimented by a different subcarrier. However, OFDM suffers from high peak to average power ratio (PAPR) due to IDFT operation at the transmitter and is sensitive to carrier-frequency and timing offsets.

Single carrier block transmission schemes as well as muticarrier systems have been widely studied in multiple user access schemes. The combination of DS-CDMA and multicarrier modulation was proposed circa 1993 [9], [10]. Since, the idea of integrating the merits of both CDMA and OFDM schemes has attracted significant research interest [11], [12], [13]. Such systems are known as Multicarrier CDMA systems and are based on some combination of DS spreading and OFDM transmission. This paper focuses on MC CDMA (Multicarrier Code Division Multiple Access) [9] and MC DS-CDMA (Multicarrier Direct Sequence Code Division Multiple Access) [10] systems incorporating Cyclic Prefix (CP) guard interval, an elegant solution which enjoys detection devoid of interblock interference (IBI) [14].

MC CDMA divides the available bandwidth into a large number of narrow orthogonal subchannels and spreads each user's data symbol in the frequency domain by transmitting all the chips of a spread symbol, at the same time, over the available subchannels [15], [16] using an OFDM-like transmission. In this context, the gain of each subchannel is different from the others. This leads to the loss of orthogonality between different users and degraded overall system performance due to MAI. MAI mitigation schemes at the receiver side include acurrate channel impairments estimation and equalisation.

On the other hand, MC DS-CDMA spread user's data symbols in the time domain. The MC DS-CDMA signal transmitted by each user's mobile station can be viewed as a set of parallel usual (single carrier) DS-CDMA signals, where all subcarriers are mutually orthogonal, and the same spreading code is used for each carrier. If the number of subcarriers is set to one, MC DS-CDMA reduces to classical DS-CDMA. Code orthogonality between users is preserved at the receiver side despite propagation through a frequencyselective multipath channel, and coherent symbol detection entails accurate channel estimation.

Since for coherent detection channel state information (CSI) is required, development of accurate channel estimation techniques are of crucial importance. Channel estimation tech- 
niques can be divided into two major categories: supervised or blind. In supervised techniques pilot symbols must be periodically transmitted in order for the receiver to acquire CSI and effectively track the channel variations. Thus, there is a tradeoff between channel estimation accuracy and data throughput. Channel coefficients can be estimated through two-dimensional linear filtering [17], [18], [19]. Estimates of the channel impulse response can also be obtained by interpolation, through adaptive schemes [20] and using decisiondirected techniques in the frequency domain [21]. Optimal location of pilots is the subject of extensive research [22], [23], [24], [25], [26]. On the other hand, blind approaches explore some knowledge of statistical characteristics of the received signal rather than information extracted from pilots. This allows for increased throughput, although blind algorithms are in general more computationally demanding than assisted ones [27] (and references therein).

This paper addresses the problem of pilot-assisted estimation of time-invariant channels in MC CDMA and MC DS-CDMA based wireless communications systems. Performances of two assisted discrete-time channel estimators, in terms of normalised mean square error (NMSE), are investigated: a conventional estimator which is based on the minimum mean square error (MMSE) criterion, and an improved estimator which exploits subspace relationships between the frequency and impulse responses of the discrete channel. In fact, the simple yet improved channel estimator introduced in [28] is based on the idea of retaining the most significant taps of the channel impulse response. In this paper, in order to investigate and compare the performance of these estimators, we reformulate the improved estimator [28] in light of a subspace projection approach [29], [30], [31], [32].

Our contributions are listed next. The subspace projection approach allows us to nicely derive analytical expressions for the NMSE of both estimators for both systems. For MC CDMA systems, we propose upper and lower bounds on the NMSE of both estimators. It is shown that for the conventional estimator, upper and lower bounds degenerate into the same expression, thus resulting in a simple closed-form solution. For the particular case of identically distributed discrete channels with common arbitrary power delay profile and Rayleigh weights, closed-form solutions also result. For MC DS-CDMA systems, we obtain simple closed-form expressions for the NMSE of both estimators.

This paper is organised as follows: the discrete signals models for MC CDMA and MC DS-CDMA systems are described in section II. Sections III and IV present, for MC CDMA and MC DS-CDMA respectively, the analysis of the pilot-aided channel estimators and equalisers used. Numerical results are presented in Section V, and Section VI draws the conclusions.

Notation: Capital boldface characters denote matrices; small boldface characters denote vectors, sometimes with subscript $M$ or $P$ to emphasise their size. The operators $(.)^{T},(.)^{H}$, $(.)^{*}$ denote transpose, Hermitian and complex conjugate, respectively, and $(\cdot)^{-1}$ denotes matrix inversion. The trace of a matrix $\mathbf{A}$ is denoted $\operatorname{tr}(\mathbf{A})$. The operator $\mathbf{E}[\cdot]$ stands for ensemble averaging, and $\mathbf{I}_{M}$ denotes the $M \times M$ identity matrix.

\section{Multicarrier CDMA System MOdel}

We consider synchronous uplink transmissions in a cell where $K$ users are active. These users employ orthogonal spreading sequences of length $N$ and their transmitted data symbols are drawn from a (2-D) complex signal constellation. Data symbols originating from different users are assumed statistically independent. The discrete channel linking the $k$ th user's transmitter to the base station, $\mathbf{h}_{k}$, is modelled as a time-invariant FIR filter with $L$ taps, that is: $\mathbf{h}_{k}=$ $\left[\begin{array}{lll}h_{k_{0}} & \ldots & h_{k_{L-1}}\end{array}\right]^{T}$; the tap gains are samples of the continous equivalent baseband complex channel impulse response. It is assumed that uplink channels remain fixed during each symbol interval. It is also assumed that a cyclic prefix (CP) of sufficient length $G_{I}$, i.e. $G_{I} \geq L-1$, is inserted before the transmission of each block in order to help eliminate interblock interference after processing at the receiver side. In the following, the discrete signals models for multicarrier CDMA (MC CDMA) and multicarrier DS-CDMA (MC DSCDMA) systems are described, respectively.

\section{A. MC CDMA System}

Figure 1 depicts the MC CDMA system at hand. The MC CDMA transmitter of the $k$-th user spreads, by a factor $N$, the original MC CDMA data symbols stream $\left\{b_{k}(i)\right\}$ in the frequency domain using a code sequence $\mathbf{c}_{k}=$ $\left[c_{k, 0}, \ldots c_{k, N-1}\right]^{T}$, with constant modulus $\left|c_{k, j}\right|^{2}=1 / N$, $\forall j$. The chips are then serial-to-parallel converted, forming an $M \times 1$ block $b_{k}(i) \mathbf{c}_{k}$ of chips which is further processed by an OFDM-like modulator using the $M$-point normalised IDFT. Without loss of generality, we assume one MC CDMA data symbol is transmitted per OFDM symbol, that is $M=N$ is assumed. The normalised IDFT is represented by a matrix $\mathbf{F}_{M, M}^{H}$, such that $\mathbf{F}_{M, M}^{H} \mathbf{F}_{M, M}$ and $\mathbf{F}_{M, M} \mathbf{F}_{M, M}^{H}$ both reduce to the identity matrix. After insertion of a $\mathrm{CP}$ of length $G_{I}$, the resulting block of dimension $P=M+G_{I}$ is transmitted through the discrete multipath channel linking the $k$-th user to the base station, $\mathbf{h}_{k}$. The transmission through $\mathbf{h}_{k}$ can be expressed employing a $P \times P$ lower triangular Toeplitz linear convolution matrix $\mathbf{H}_{k}$, with first column: $\left[\begin{array}{llllll}h_{k_{0}} & \ldots & h_{k_{L-1}} & 0 & \ldots & 0\end{array}\right]^{T}$ with $\mathrm{E}\left[\left\|\mathbf{h}_{k}\right\|^{2}\right]=1$ for normalization purposes.

At the base station, the $\mathrm{CP}$ is removed from the received block, which turns the channel linear convolution into a circular one. Further processing involves the application of an $M$-point DFT to the resulting block, yielding the received signal at the base station:

$$
\mathbf{r}(i)=\sum_{k=1}^{K} \mathbf{C}_{k} \mathbf{q}_{k} b_{k}(i)+\mathbf{n}(i)=\sum_{k=1}^{K} \mathbf{Q}_{k} \mathbf{c}_{k} b_{k}(i)+\mathbf{n}(i),
$$

where $\mathbf{C}_{k}=\operatorname{diag}\left(\mathbf{c}_{k}\right)$ is a $M \times M$ diagonal matrix, whose elements are the chips of the spreading sequence of the $k$-th user; $\mathbf{n}(i)$ is a zero-mean complex white Gaussian noise vector with covariance matrix $\mathrm{E}\left[\mathbf{n}(i) \mathbf{n}^{H}(i)\right]=\sigma^{2} \mathbf{I}_{M} ; b_{k}(i)$ is the $i$-th data symbol transmitted by the $k$-th user with $\left|b_{k}(i)\right|=1$ and $\mathrm{E}\left[b_{k}(i)\right]=0$. 


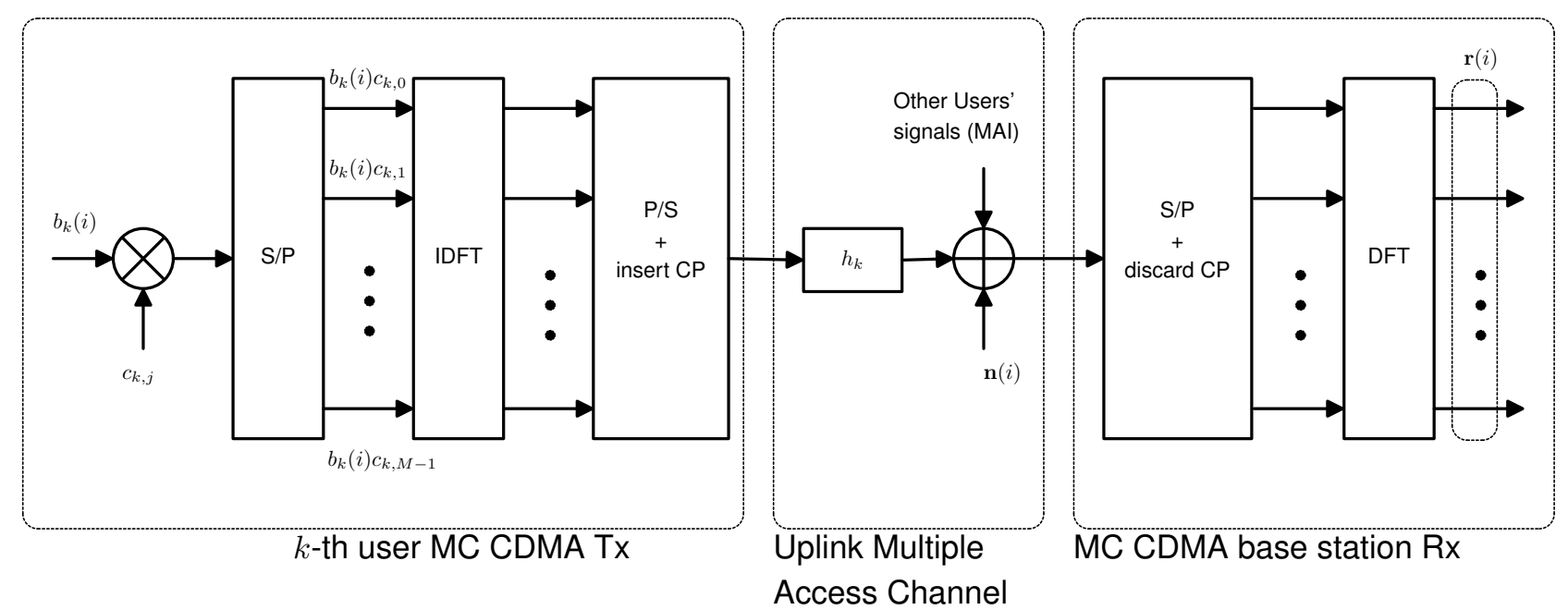

Fig. 1. Block diagram of MC CDMA system

In (1), $\mathbf{Q}_{k}$ is an $M \times M$ diagonal matrix, whose entries contain the elements of the discrete channel frequency response, given by the $M \times 1$ vector:

$$
\mathbf{q}_{k}=\sqrt{M} \mathbf{F}_{M, M} \mathbf{h}_{k_{e x t}}
$$

where $\mathbf{h}_{k_{e x t}}=\left[h_{k_{0}} \ldots h_{k_{L-1}} 0 \ldots 0\right]$ is the channel impulse response $\mathbf{h}_{k}$ padded with $M-L$ zeros; thus, $\mathrm{E}\left[\left\|\mathbf{h}_{k}\right\|^{2}\right]=1$ and $\mathrm{E}\left[\left\|\mathbf{q}_{k}\right\|^{2}\right]=M$.

The channel frequency response $\mathbf{q}_{k}$ in (2) can also be written in terms of the $L \times 1$ channel impulse response vector $\mathbf{h}_{k}$ :

$$
\mathbf{q}_{k}=\sqrt{M} \mathbf{F}_{M, L} \mathbf{h}_{k}
$$

where $\mathbf{F}_{M, L}$ is a $M \times L$ matrix containing the first $L$ columns of an $M$-point DFT matrix.

\section{B. MC DS-CDMA System}

In MC DS-CDMA, depicted in Figure 2 , the transmitter of the $k$-th user performs a serial-to-parallel conversion on the data symbols sequence, forming data blocks $\mathbf{b}_{k}(i)$ of length $M$. The symbols in each data block $\mathbf{b}_{k}(i)$ are spread by the same length $N$ spreading sequence and transmitted chipby-chip using an OFDM scheme. The length $M$ 'frequencydomain' symbol fed to the OFDM transmitter $k$ during the $j$-th chip signalling interval $(j=0,1, \ldots, N-1)$ of the $i$-th data block $\mathbf{b}_{k}(i)$ is given by $\mathbf{b}_{k}(i) c_{k, j}$, where $c_{k, j}$ is the $j$-th chip of the spreading sequence assigned to the $k$-th user. After cyclic-prefix insertion, the resulting OFDM symbol of length $P=M+G_{I}$ is transmitted through the discrete multipath channel $\mathbf{h}_{k}$.

The MC DS-CDMA receiver at the base station removes the cyclic prefix and computes the $M$-point DFT of the resulting OFDM symbol. The received signal is the superposition of the $K$ signals corresponding to the transmission of the $j$-th chip of the $i$-th data block of the $k$-th user, corrupted by noise:

$$
\mathbf{x}(i ; j)=\sum_{k=1}^{K} \mathbf{Q}_{k} \mathbf{b}_{k}(i) c_{k, j}+\mathbf{n}(i ; j) ; j=0,1, \ldots, N-1
$$

where $\mathbf{Q}_{k}=\operatorname{diag}\left(\mathbf{q}_{k}\right)$ with $\mathbf{q}_{k}$ as in (3), and $\mathbf{n}(i ; j)$ is a zeromean complex white Gaussian noise vector with covariance matrix $\mathrm{E}\left[\mathbf{n}(i ; j) \mathbf{n}^{H}(i ; j)\right]=\sigma^{2} \mathbf{I}_{M}$.

Collecting $N$ consecutive chip blocks, an $M \times N$ matrix for the $i$-th data symbol block can be written as:

$$
\begin{aligned}
\mathbf{X}(i) & =[\mathbf{x}(i, 0) \ldots, \mathbf{x}(i, N-1)] \\
& =\sum_{k=1}^{K} \mathbf{Q}_{k} \mathbf{b}_{k}(i) \mathbf{c}_{k}^{T}+\mathbf{N}(i)
\end{aligned}
$$

where $\mathbf{N}(i)=[\mathbf{n}(i ; 0) \ldots, \mathbf{n}(i ; N-1)]$.

\section{Channel Estimation And Equalis ATion For MC CDMA}

Let $m$ be the index of the user of interest. The conventional channel estimation scheme used here for MC CDMA is based on the MMSE criterion as in [33]. Accordingly,

$$
\widehat{\mathbf{q}}_{m}^{(m m s e)}=\arg \min _{\mathbf{q}} \mathbf{E}\left[\left\|\mathbf{r}(i)-\mathbf{C}_{m} \mathbf{q} b_{m}(i)\right\|^{2}\right],
$$

whose solution is an unbiased channel estimate which would indeed lead to the true channel frequency response:

$$
\widehat{\mathbf{q}}_{m}^{(m m s e)}=\mathbf{C}_{m}^{-1} \mathrm{E}\left[\mathbf{r}(i) b_{m}^{*}(i)\right]=\mathbf{q}_{m} .
$$

The usual approach of approximating the expectation by an average over $N_{p}$ pilot symbols yields the unbiased estimator given by:

$$
\widehat{\mathbf{q}}_{m}=\frac{\mathbf{C}_{m}^{-1}}{N_{p}} \sum_{i=1}^{N_{p}} \mathbf{r}(i) b_{m}^{*}(i)
$$

\section{A. Improved Channel Estimator}

The channel estimate resulting from (8) can be improved thanks to the fact that, in general, the channel impulse response length $L$ satisfies $L \leq M$. As a result, any component $\widehat{h}_{m}(l), L \leq l<M$, in a given channel impulse response estimate is considered noisy (i.e. non-significant tap) and can be removed [28]. The improved estimation procedure consists in: i) applying an $M$-point IDFT to the channel 


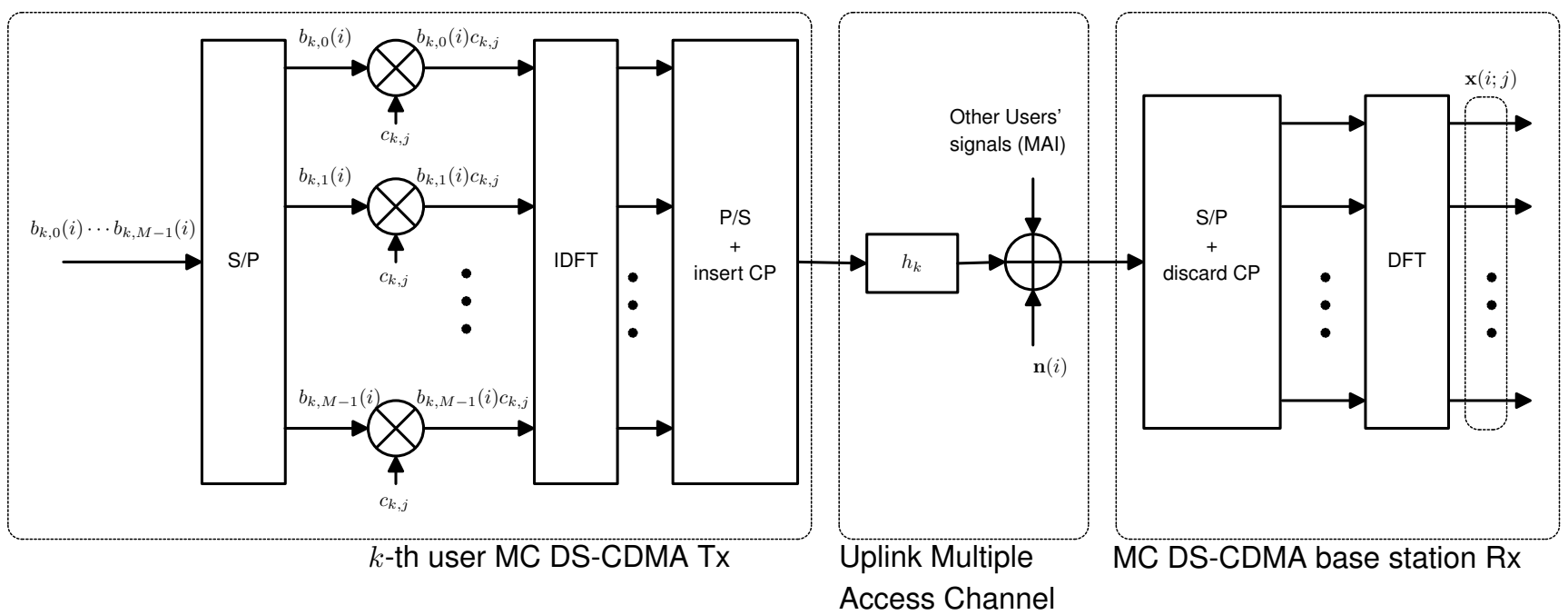

Fig. 2. Block diagram of MC DS-CDMA system

frequency response estimate in order to obtain the channel impulse response estimate, ii) then replace the last $(M-L)$ elements of this impulse response by zeros, and iii) transform back the remaining coefficients, called significants taps, to the frequency domain, applying an $M$-Point DFT. After this last step, an improved channel frequency response estimate $\widehat{\mathbf{q}}_{m}^{w}$ results. Thus, the improved estimator is obtained as follows:

$\widehat{\mathbf{q}}_{m} \stackrel{I D F T}{\longrightarrow}\left[\begin{array}{c}\widehat{h}_{m}(0) \\ \vdots \\ \widehat{h}_{m}(L-1) \\ \widehat{h}_{m}(L) \\ \vdots \\ \widehat{h}_{m}(M-1)\end{array}\right] \longrightarrow\left[\begin{array}{c}\widehat{h}_{m}(0) \\ \vdots \\ \widehat{h}_{m}(L-1) \\ 0 \\ \vdots \\ 0\end{array}\right] \stackrel{D F T}{\longrightarrow} \widehat{\mathbf{q}}_{m}^{w}$.

Looking from a different perspective [29], [30], [31], [32], it can be shown that this procedure is equivalent to projecting the original channel frequency response estimate in the subspace generated by the first $L$ columns of the DFT matrix $\mathbf{F}_{M, M}$. Note that by virtue of (3) the true frequency response of the channel has to lie in this subspace. The true value of $L$ is in general not known a priori, and one can make the conservative assumption $\hat{L}=G=G_{I}+1$ since $L-1 \leq G_{I}$. Then, the true channel frequency response (CFR) also lies in the subspace spanned by the first $G$ columns of $\mathbf{F}_{M, M}$. The projection matrix $\mathbf{W}_{o}$ that projects a length $M$ vector onto the subspace spanned by the first $G$ columns of $\mathbf{F}_{M, M}$ is given by

$$
\mathbf{W}_{o}=\mathbf{F}_{M, G}\left(\mathbf{F}_{M, G}^{H} \mathbf{F}_{M, G}\right)^{-1} \mathbf{F}_{M, G}^{H},
$$

where $\mathbf{F}_{M, G}$ is a $M \times G$ matrix containing the first $G$ columns of the $M$-point DFT matrix. Using the fact that $\mathbf{F}_{M, G}^{H} \mathbf{F}_{M, G}=$ $\mathbf{I}_{G}$, we have

$$
\mathbf{W}_{o}=\mathbf{F}_{M, G} \mathbf{F}_{M, G}^{H} .
$$

Hence, the improved channel estimate is also an unbiased estimate and is given by:

$$
\widehat{\mathbf{q}}_{m}^{w}=\mathbf{W}_{o} \widehat{\mathbf{q}}_{m}=\mathbf{W}_{o} \mathbf{C}_{m}^{-1} \frac{1}{N_{p}} \sum_{i=1}^{N_{p}} \mathbf{r}(i) b_{m}^{*}(i) .
$$

It should be stressed that no matter how the original channel frequency response estimate $\widehat{\mathbf{q}}_{m}$ is obtained, the use of the projection matrix $\mathbf{W}_{o}$ leads to a reduced estimation error (see appendix); in this regard, the subspace projection procedure on its own can be viewed as a channel estimate improver. The performance enhancement, however, affects differently the noise and multiple access interference (MAI) components of the estimation error, as will be seen shortly.

\section{B. Statistical Analysis}

In the following, the performances of the conventional and improved estimators are derived. In order to address both cases, consider an $M \times M$ matrix $\mathbf{A}_{m}$ which evaluates to 1) $\mathbf{A}_{m}=\mathbf{C}_{m}^{-1}$ in (8) and 2) $\mathbf{A}_{m}=\mathbf{W}_{o} \mathbf{C}_{m}^{-1}$ in (9), both leading to unbiased estimators since $\mathbf{A}_{m} \mathbf{C}_{m} \mathbf{q}_{m}=\mathbf{q}_{m}$.

The channel estimator of the $m$-th user can be written as:

$$
\widehat{\mathbf{q}}_{m}=\mathbf{q}_{m}+\mathbf{A}_{m} \frac{1}{N_{p}} \sum_{\substack{k=1 \\ k \neq m}}^{K} \mathbf{C}_{k} \mathbf{q}_{k} \mathbf{b}_{m}^{H} \mathbf{b}_{k}+\mathbf{A}_{m} \frac{1}{N_{p}} \sum_{i=1}^{N_{p}} \mathbf{n}(i) b_{m}^{*}(i),
$$

where $\mathbf{b}_{m}^{H} \mathbf{b}_{k}=\sum_{i=1}^{N_{p}} b_{m}^{*}(i) b_{k}(i)$, with the vector $\mathbf{b}_{m}=$ $\left[b_{m}(1) \ldots b_{m}\left(N_{p}\right)\right]^{T}$.

The mean square error (MSE) of the channel estimator of the $m$-th user, given the $K$ channels, is:

$$
\varepsilon_{m}^{2}=\mathrm{E}\left[\left\|\widehat{\mathbf{q}}_{m}-\mathbf{q}_{m}\right\|^{2} \mid \mathbf{q}_{1}, \mathbf{q}_{2}, \ldots, \mathbf{q}_{K}\right],
$$

which, after some manipulation, results in

$$
\begin{aligned}
\varepsilon_{m}^{2}= & \frac{1}{N_{p}} \sum_{\substack{k=1 \\
k \neq m}}^{K} \sum_{\substack{t=1 \\
t \neq m}}^{K} \mathbf{q}_{k}^{H} \mathbf{C}_{k}^{H}\left(\mathbf{A}_{m}^{H} \mathbf{A}_{m}\right) \mathbf{C}_{t} \mathbf{q}_{t} \mathrm{E}\left[\mathbf{b}_{k}^{H} \mathbf{b}_{m} \mathbf{b}_{m}^{H} \mathbf{b}_{t}\right] \\
& +\frac{\sigma^{2}}{N_{p}} \operatorname{tr}\left[\mathbf{A}_{m} \mathbf{A}_{m}^{H}\right] .
\end{aligned}
$$

Since the symbols of different users are assumed to be statistically independent, the expectation in (12) reduces to:

$$
\mathrm{E}\left[\mathbf{b}_{m}^{H} \mathbf{b}_{k} \mathbf{b}_{t}^{H} \mathbf{b}_{m}\right]=\left\{\begin{array}{ccc}
N_{p}, & \text { if } & k=t \\
0, & \text { if } & k \neq t
\end{array} .\right.
$$


It turns out that (12) can be further simplified to:

$$
\varepsilon_{m}^{2}=\frac{1}{N_{p}} \sum_{k=1, k \neq m}^{K} \mathbf{q}_{k}^{H} \mathbf{C}_{k}^{H} \mathbf{A}_{m}^{H} \mathbf{A}_{m} \mathbf{C}_{k} \mathbf{q}_{k}+\frac{\sigma^{2}}{N_{p}} \operatorname{tr}\left[\mathbf{A}_{m} \mathbf{A}_{m}^{H}\right] .
$$

Thus,

$$
\begin{aligned}
\varepsilon_{m}^{2} & =\frac{1}{N_{p}}[\sum_{k=1}^{K} \mathbf{q}_{k}^{H} \mathbf{C}_{k}^{H} \mathbf{A}_{m}^{H} \mathbf{A}_{m} \mathbf{C}_{k} \mathbf{q}_{k}-\underbrace{\mathbf{q}_{m}^{H} \mathbf{C}_{m}^{H} \mathbf{A}_{m}^{H} \mathbf{A}_{m} \mathbf{C}_{m} \mathbf{q}_{m}}_{\left\|\mathbf{q}_{m}\right\|^{2}}] \\
& +\frac{\sigma^{2}}{N_{p}} \operatorname{tr}\left[\mathbf{A}_{m} \mathbf{A}_{m}^{H}\right] .
\end{aligned}
$$

Since $\mathbf{A}_{m} \mathbf{C}_{m} \mathbf{q}_{m}=\mathbf{q}_{m}$ and considering $K$ active users, the average MSE over all users can be written as:

$$
\begin{aligned}
\varepsilon^{2}= & \frac{1}{K} \sum_{m=1}^{K} \varepsilon_{m}^{2} \\
= & {\left[\frac{1}{N_{p}} \sum_{k=1}^{K} \mathbf{q}_{k}^{H} \mathbf{C}_{k}^{H} \mathbf{B} \mathbf{C}_{k} \mathbf{q}_{k}-\frac{1}{K} \sum_{m=1}^{K}\left\|\mathbf{q}_{m}\right\|^{2}\right] } \\
& +\frac{\sigma^{2}}{N_{p}} \operatorname{tr}(\mathbf{B})
\end{aligned}
$$

where

$$
\mathbf{B}=\frac{1}{K} \sum_{m=1}^{K} \mathbf{A}_{m} \mathbf{A}_{m}^{H}
$$

To proceed further, it is assumed that the channels of the different users are: $i$ ) normalised with $\mathrm{E}\left[\left\|\mathbf{h}_{k}\right\|^{2}\right]=1$ and hence $\mathrm{E}\left[\left\|\mathbf{q}_{k}\right\|^{2}\right]=M$, and $i$ ) identically distributed. Thus, without loss of generality, the statistics of the channel frequency response of any given user, say the first user, can be used. The expectation of (13), i.e., $\epsilon^{2}=\mathrm{E}\left[\varepsilon^{2}\right]$, leads to:

$$
\epsilon^{2}=\frac{1}{N_{p}}\left[\mathrm{E}\left[\mathbf{q}_{1}^{H} \mathbf{D}_{o} \mathbf{q}_{1}\right]-M\right]+\frac{\sigma^{2}}{N_{p}} \operatorname{tr}(\mathbf{B}),
$$

where matrix $\mathbf{D}_{o}=\sum_{k=1}^{K} \mathbf{C}_{k}^{H} \mathbf{B} \mathbf{C}_{k}$.

Finally, the normalised mean square error (NMSE) averaged over the $K$ users is given by $e^{2}=\frac{\epsilon^{2}}{M}$ :

$$
e^{2}=\frac{1}{M N_{p}}\left[\mathrm{E}\left[\mathbf{q}_{1}^{H} \mathbf{D}_{o} \mathbf{q}_{1}\right]-M\right]+\frac{\sigma^{2}}{M N_{p}} \operatorname{tr}(\mathbf{B}) .
$$

Expressing (16) in terms of the channel impulse response $\mathbf{q}_{1}=\sqrt{M} \mathbf{F}_{M, G} \mathbf{h}_{1}$ yields:

$$
\begin{aligned}
e^{2} & =\frac{1}{N_{p}}\left[\mathrm{E}\left[\mathbf{h}_{1}^{H} \mathbf{F}_{M, G}^{H} \mathbf{D}_{o} \mathbf{F}_{M, G} \mathbf{h}_{1}\right]-1\right]+\frac{\sigma^{2}}{M N_{p}} \operatorname{tr}(\mathbf{B}) \\
& =\frac{1}{N_{p}}\left[\mathrm{E}\left[\mathbf{h}_{1}^{H} \mathbf{D}_{F} \mathbf{h}_{1}\right]-1\right]+\frac{\sigma^{2}}{M N_{p}} \operatorname{tr}(\mathbf{B})
\end{aligned}
$$

where

$$
\mathbf{D}_{F}=\mathbf{F}_{M, G}^{H} \mathbf{D}_{o} \mathbf{F}_{M, G}=\mathbf{F}_{M, G}^{H}\left(\sum_{k=1}^{K} \mathbf{C}_{k}^{H} \mathbf{B C}_{k}\right) \mathbf{F}_{M, G}
$$

It is possible to upper and lower bound the normalised mean square error $e^{2}$ in (17) by carrying out an Eigenvalue decomposition (EVD) in order to obtain the minimum and maximum eigenvalues, $\lambda_{\min }$ and $\lambda_{\max }$ respectively, of the $G \times G$ matrix $\mathbf{D}_{F}$ in (18):

$$
\left\|\mathbf{h}_{1}\right\|^{2} \lambda_{\min } \leq \mathbf{h}_{1}^{H} \mathbf{D}_{F} \mathbf{h}_{1} \leq \lambda_{\max }\left\|\mathbf{h}_{1}\right\|^{2} .
$$

Taking expectation on (19) and using $\mathrm{E}\left[\left\|\mathbf{h}_{1}\right\|^{2}\right]=1$ yields:

$$
\begin{aligned}
\mathrm{E}\left[\left\|\mathbf{h}_{1}\right\|^{2}\right] \lambda_{\min } & \leq \mathrm{E}\left[\mathbf{h}_{1}^{H} \mathbf{D}_{F} \mathbf{h}_{1}\right] \leq \lambda_{\max } \mathrm{E}\left[\left\|\mathbf{h}_{1}\right\|^{2}\right] \\
\lambda_{\min } & \leq \mathrm{E}\left[\mathbf{h}_{1}^{H} \mathbf{D}_{F} \mathbf{h}_{1}\right] \leq \lambda_{\max }
\end{aligned}
$$

Thus, the lower and upper bounds of the NMSE of the channel estimator in (17) are

$$
\frac{1}{N_{p}}\left[\lambda_{\min }-1\right]+\frac{\sigma^{2}}{M N_{p}} \operatorname{tr}(\mathbf{B}) \leq e^{2} \leq \frac{1}{N_{p}}\left[\lambda_{\max }-1\right]+\frac{\sigma^{2}}{M N_{p}} \operatorname{tr}(\mathbf{B}) .
$$

It should be emphasised that the bounds in (21) are general and independent of the channel characteristics, given the assumptions made.

To proceed further, the conventional estimator (case $\mathbf{A}_{\mathbf{m}}=$ $\mathbf{C}_{m}^{-1}$ ) and the improved estimator (case $\mathbf{A}_{m}=\mathbf{W}_{o} \mathbf{C}_{m}^{-1}$ ) must be addressed separately.

1) Case $\mathbf{A}_{\mathbf{m}}=\mathbf{C}_{m}^{-1}$ : Consider the channel estimator in (10) with $\mathbf{A}_{m}=\mathbf{C}_{m}^{-1}$ which reduces to the conventional estimator in (8). The matrices $\mathbf{B}$ in (14) and $\mathbf{D}_{F}$ in (18) evaluate to

$$
\mathbf{B}=\frac{1}{K} \sum_{m=1}^{K} \mathbf{C}_{m}^{-1} \mathbf{C}_{m}^{-1 H}
$$

and

$$
\begin{aligned}
\mathbf{D}_{F} & =\mathbf{F}_{M, G}^{H} \sum_{k=1}^{K} \operatorname{diag}\left(\mathbf{c}_{k}^{*}\right) \mathbf{B} \operatorname{diag}\left(\mathbf{c}_{k}\right) \mathbf{F}_{M, G} \\
& =\mathbf{F}_{M, G}^{H}\left(\sum_{k=1}^{K} \mathbf{c}_{k}^{*} \mathbf{c}_{k}^{T} \odot \mathbf{B}\right) \mathbf{F}_{M, G} \\
& =\mathbf{F}_{M, G}^{H}(\mathcal{C} \odot \mathbf{B}) \mathbf{F}_{M, G},
\end{aligned}
$$

where $\mathcal{C}=\sum_{m=1}^{K} \mathbf{c}_{m}^{*} \mathbf{c}_{m}^{T}$, and $\odot$ stands for the Hadamard product.

Since the spreading codes are assumed to be normalised, $\mathbf{C}_{m}^{-1} \mathbf{C}_{m}^{-1 H}=M \mathbf{I}_{M, M}$ and $\mathbf{C}_{k}^{H} \mathbf{C}_{k}=\frac{1}{M} \mathbf{I}_{M, M}$ hold. Then, matrix $\mathbf{B}$ in (22) further reduces to:

$$
\mathbf{B}=\mathbf{B}_{c}=\mathbf{C}_{m}^{-1} \mathbf{C}_{m}^{-1 H}=M \mathbf{I}_{M, M},
$$

and its trace is given by:

$$
\operatorname{tr}(\mathbf{B})=\operatorname{tr}\left(\mathbf{B}_{c}\right)=M \operatorname{tr}\left(\mathbf{I}_{M, M}\right)=M^{2} .
$$

Matrix $\mathbf{D}_{F}$ in (23) becomes:

$$
\mathbf{D}_{F}=\mathbf{D}_{F}^{c}=K \mathbf{F}_{M, G}^{H} \mathbf{F}_{M, G}=K \mathbf{I}_{G, G}
$$

Eigenvalue decompositon of $\mathbf{D}_{F}$ in (26) for the conventional estimator case leads to $\lambda_{\max }=\lambda_{\min }=K$. Applying these results to (21), the NMSE of the conventional estimator $\left(e_{c}^{2}\right)$ boils down to:

$$
e_{c}^{2}=\frac{1}{N_{p}}[K-1]+\frac{M \sigma^{2}}{N_{p}} .
$$

It is interesting to note that the equality in (27) for the conventional estimator in (8) does not depend on the users' channel characteristics; it is only dependent on the number of active users $K$, the number of carriers used $M$, the number of transmitted pilot symbols $N_{p}$, and the noise power $\sigma^{2}$. 
2) Case $\mathbf{A}_{m}=\mathbf{W}_{o} \mathbf{C}_{m}^{-1}$ : Now consider the channel estimator in (10) with $\mathbf{A}_{m}=\mathbf{W}_{o} \mathbf{C}_{m}^{-1}$ which leads to the improved estimator in (9).

Using the fact that $\mathbf{W}_{o}$, as a projection matrix, satisfies $\mathbf{W}_{o}^{H} \mathbf{W}_{o}=\mathbf{W}_{o} \mathbf{W}_{o}^{H}=\mathbf{W}_{o}$, then matrix $\mathbf{B}$ in (14) now becomes:

$$
\begin{aligned}
\mathbf{B}=\mathbf{B}_{w} & =\frac{1}{K} \sum_{m=1}^{K} \mathbf{C}_{m}^{-1} \mathbf{W}_{o} \mathbf{C}_{m}^{-1 H} \\
& =\frac{1}{K} \sum_{m=1}^{K} \operatorname{diag}\left(\mathbf{c}_{m}^{\prime}\right) \mathbf{W}_{o} \operatorname{diag}\left(\mathbf{c}_{m}^{\prime *}\right) \\
& =\frac{1}{K} \sum_{m=1}^{K} \mathbf{c}_{m}^{\prime} \mathbf{c}_{m}^{\prime} \odot \mathbf{W}_{o} \\
& =\frac{1}{K}\left(\mathcal{C}^{\prime} \odot \mathbf{W}_{o}\right),
\end{aligned}
$$

where matrix $\mathcal{C}^{\prime}=\sum_{m=1}^{K} \mathbf{c}_{m}^{\prime} \mathbf{c}_{m}^{\prime}$, with $\mathbf{c}_{k}^{\prime}=$ $\left[1 / c_{k, 0}, \ldots 1 / c_{k, M-1}\right]^{T}$. And, using the fact that $\operatorname{tr}\left(\mathbf{W}_{o}\right)=\frac{G}{M}$ :

$$
\begin{aligned}
\operatorname{tr}(\mathbf{B})=\operatorname{tr}\left(\mathbf{B}_{w}\right) & =\frac{1}{K} \operatorname{tr}\left(\mathcal{C}^{\prime} \odot \mathbf{W}_{o}\right) \\
& =\frac{1}{K} K M \operatorname{tr}\left[\mathbf{W}_{o}\right]=M \frac{G}{M}=G .
\end{aligned}
$$

Matrix $\mathbf{D}_{F}$ in (18) becomes:

$$
\begin{aligned}
\mathbf{D}_{F}=\mathbf{D}_{F}^{w} & =\mathbf{F}_{M, G}^{H} \sum_{k=1}^{K} \mathbf{C}_{k}^{H} \mathbf{B}_{w} \mathbf{C}_{k} \mathbf{F}_{M, G} \\
& =\mathbf{F}_{M, G}^{H} \sum_{k=1}^{K} \mathbf{C}_{k}^{H}\left(\frac{1}{K}\left(\mathcal{C}^{\prime} \odot \mathbf{W}_{o}\right)\right) \mathbf{C}_{k} \mathbf{F}_{M, G} \\
& =\frac{1}{K} \mathbf{F}_{M, G}^{H}\left(\mathcal{C} \odot \mathcal{C}^{\prime} \odot \mathbf{W}_{o}\right) \mathbf{F}_{M, G}
\end{aligned}
$$

Applying (29) and (30) to (17), the NMSE of the improved channel estimator $\left(e_{w}^{2}\right)$ is obtained as

$$
e_{w}^{2}=\frac{1}{N_{p}}\left[\mathrm{E}\left[\mathbf{h}_{1}^{H} \mathbf{D}_{F}^{w} \mathbf{h}_{1}\right]-1\right]+\frac{G \sigma^{2}}{N_{p}} .
$$

The lower and upper bounds of $e_{w}^{2}$ are in turn given by:

$$
\frac{1}{N_{p}}\left[\lambda_{\min }-1\right]+\frac{G \sigma^{2}}{N_{p}} \leq e_{w}^{2} \leq \frac{1}{N_{p}}\left[\lambda_{\max }-1\right]+\frac{G \sigma^{2}}{N_{p}},
$$

where $\lambda_{\min }$ and $\lambda_{\max }$ are the minimum and maximum eigenvalues of $\mathbf{D}_{F}^{w}$ as defined in (30), respectively.

The comparison of (27) and (31) motivates the defintion of the MAI reduction factor $\eta$ as:

$$
\eta=\frac{1}{K-1} \mathrm{E}\left[\left[\mathbf{h}_{1}^{H} \mathbf{D}_{F}^{w} \mathbf{h}_{1}\right]-1\right]
$$

and the noise reduction factor $\gamma$ as:

$$
\gamma=\frac{\operatorname{tr}\left(\mathbf{B}_{w}\right)}{\operatorname{tr}(\mathbf{B})}=\frac{G}{M} .
$$

In (34), $\gamma=\frac{G}{M}<1$, thus confirming the reduction of the noise component contribution to the estimation error.
It is also possible to express the lower and upper bounds on $e_{w}^{2}$ in terms of the newly defined factors $\eta$ and $\gamma$ as:

$$
\frac{K-1}{N_{p}} \eta_{\min }+\gamma \frac{M \sigma^{2}}{N_{p}} \leq e_{w}^{2} \leq \frac{K-1}{N_{p}} \eta_{\max }+\gamma \frac{M \sigma^{2}}{N_{p}},
$$

with $\eta_{\min }$ and $\eta_{\max }$ given, respectively, by :

$$
\eta_{\min }=\frac{\lambda_{\min }-1}{K-1}, \quad \text { and } \quad \eta_{\max }=\frac{\lambda_{\max }-1}{K-1} .
$$

A Particular Case - Channel with uncorrelated coefficients and arbitrary power delay profile: Now let the channel coefficients be $h_{k_{i}}=\alpha_{k_{i}} p_{i} ; \forall i=0 \ldots G-1$ where $\alpha_{k_{i}}$ are complex uncorrelated random variables with zero mean and $\mathrm{E}\left[\left|\alpha_{k_{i}}\right|^{2}\right]=1$, and the weights $p_{i}$ satisfying $\sum_{i=0}^{G-1}\left|p_{i}\right|^{2}=1$ are common to the $K$ users.

For the improved estimator, $\eta$ evaluates to :

$$
\eta=\frac{1}{K-1} \sum_{i=0}^{G-1}\left|p_{i}\right|^{2}\left(d_{i, i}^{w}-1\right)
$$

where $\left\{d_{i, j}^{w}\right\}$ are the elements of matrix $\mathbf{D}_{F}^{w}$ in (30), which depends only on DFT coefficients and on the spreading codes in use.

It follows that the NMSE of the improved estimator becomes:

$$
e_{w}^{2}=\frac{1}{N_{p}} \sum_{i=0}^{G-1}\left|p_{i}\right|^{2}\left(d_{i, i}^{w}-1\right)+\frac{\sigma^{2} G}{N_{p}}
$$

\section{Equalisation and Detection}

Employing the channel estimates obtained in the previous sections, equalisation of (1) followed by symbol detection can be performed. One possibility is to apply an MMSE equaliser:

$$
\mathbf{w}_{m}=\arg \min _{\mathbf{w}} \mathrm{E}\left[\left\|b_{m}(i)-\mathbf{w}^{H} \mathbf{r}(i)\right\|^{2}\right]
$$

whose solution is $\mathbf{w}_{m}=\mathbf{R}^{-1} \mathrm{E}\left[\mathbf{r}(i) b_{m}^{*}\right]$ where matrix $\mathbf{R}^{-1}$ is the inverse of the correlation matrix. Here, the correlation matrix is estimated using $\widehat{\mathbf{R}}(i)=\frac{1}{i} \sum_{j=1}^{i} \mathbf{r}(j) \mathbf{r}^{H}(j)$. Since $\mathbf{C}_{m}^{-1} \mathbf{E}\left[\mathbf{r}(i) b_{m}^{*}(i)\right]=\mathbf{q}_{m}$, the term $\mathbf{E}\left[\mathbf{r}(i) b_{m}^{*}(i)\right]$ appearing in $\mathbf{w}_{m}$ can be approximated by $\mathbf{C}_{m} \widehat{\mathbf{q}}_{m}$ with the estimate $\widehat{\mathbf{q}}_{m}$ given by the conventional estimate in (8), or by the improved estimate in (9).

After equalisation, the detected symbol of the $m$-th user, $b_{m}(i)$, can be obtained as $\widehat{b}_{m}(i)=\operatorname{sgn}\left(\operatorname{Re}\left[\mathbf{w}_{m}^{H} \mathbf{r}(i)\right]\right)$, for the case of BPSK modulation.

\section{Channel Estimation FOR MC DS-CDMA}

From (5) it can be seen that the code orthogonality between users is preserved despite propagation through the multipath channel. MAI is eliminated via code-matched filtering and the received signal at the base station for the $m$-th desired user is:

$$
\begin{aligned}
\mathbf{y}_{m}(i) & =\mathbf{X}(i) \mathbf{c}_{m}^{*} \\
& =\mathbf{Q}_{m} \mathbf{b}_{m}(i)+\mathbf{n}_{f}(i) \\
& =\mathbf{S}_{m}(i) \mathbf{q}_{m}+\mathbf{n}_{f}(i),
\end{aligned}
$$


where $\mathbf{S}_{m}(i)=\operatorname{diag}\left(\mathbf{b}_{m}(i)\right)$, and $\mathbf{n}_{f}(i)=\mathbf{N}(i) \mathbf{c}_{m}^{*}$ is a zeromean complex Gaussian noise vector with covariance matrix $\mathrm{E}\left[\mathbf{n}_{f}(i) \mathbf{n}_{f}^{H}(i)\right]=\left\|\mathbf{c}_{m}\right\|^{2} \sigma^{2} \mathbf{I}_{M}=\sigma^{2} \mathbf{I}_{M}$

The (conventional) channel estimator for MC DS-CDMA minimises the mean square error between the received signal $\mathbf{y}_{m}(i)$ and a reconstructed signal for user $m$ :

$$
\widehat{\mathbf{q}}_{m}^{(m m s e)}=\arg \min _{\mathbf{q}} \mathbf{E}\left[\left\|\mathbf{y}_{m}(i)-\mathbf{S}_{m}(i) \mathbf{q}\right\|^{2}\right],
$$

yielding an unbiased channel estimate (it would indeed lead to the true channel frequency response):

$$
\widehat{\mathbf{q}}_{m}^{(m m s e)}=\mathrm{E}\left[\mathbf{S}_{m}^{H}(i) \mathbf{y}_{m}(i)\right]=\mathbf{q}_{m} .
$$

Replacing the expectation term in (41) by a temporal average over $\ell$ consecutive pilot symbol blocks (number of pilot symbols satisfies $N_{p}=\ell M$ ), the channel estimate becomes:

$$
\widehat{\mathbf{q}}_{m}=\frac{1}{\ell} \sum_{i=1}^{\ell} \mathbf{S}_{m}^{H}(i) \mathbf{y}_{m}(i)=\mathbf{q}_{m}+\frac{1}{\ell} \sum_{i=1}^{\ell} \mathbf{S}_{m}^{H}(i) \mathbf{n}_{f}(i) .
$$

As MAI was removed at despreading, channel estimate errors are due only to background noise. The NMSE for conventional channel estimation in MC DS-CDMA systems then reduces to:

$$
e_{c}^{2}=\frac{\sigma^{2}}{\ell}=\frac{\sigma^{2} M}{N_{p}} .
$$

\section{A. Improved Channel Estimation}

As described in section III-A, the channel estimate in (42) can be improved by virtue of the projection matrix $\mathbf{W}_{o}$ :

$$
\widehat{\mathbf{q}}_{m}^{w}=\mathbf{W}_{o} \widehat{\mathbf{q}}_{m}=\mathbf{W}_{o} \frac{1}{\ell} \sum_{i=1}^{\ell} \mathbf{S}_{m}^{H}(i) \mathbf{y}_{m}(i) .
$$

As in the MC CDMA case, the variance of the noise induced estimation error is reduced by a factor of $G / M$. Hence

$$
e_{w}^{2}=\frac{\sigma^{2} G}{N_{p}}
$$

\section{B. Equalisation and Detection}

The MMSE equaliser is given by:

$$
\boldsymbol{\Gamma}=\left(\widehat{\mathbf{Q}}_{m} \widehat{\mathbf{Q}}_{m}^{H}+\sigma^{2} \mathbf{I}_{M}\right)^{-1} \widehat{\mathbf{Q}}_{m}
$$

where $\widehat{\mathbf{Q}}_{m}=\operatorname{diag}\left(\widehat{\mathbf{q}}_{m}\right)$, with $\widehat{\mathbf{q}}_{m}$ given by (42) or by (44). It should be noted that $\widehat{\mathbf{Q}}_{m} \widehat{\mathbf{Q}}_{m}^{H}$ and thus $\boldsymbol{\Gamma}$ in (46) are diagonal matrices.

After equalisation, the detected symbol of the $m$-th user, $\widehat{\mathbf{b}}_{m}(i)$, can be obtained as $\widehat{\mathbf{b}}_{m}(i)=\operatorname{sgn}\left(\operatorname{Re}\left[\boldsymbol{\Gamma}^{H} \mathbf{y}_{m}(i)\right]\right)$.

We remark that since in the MC DS-CDMA case the signals of the users can be decoupled, MMSE equalisation and detection of any given user can be performed once the channel of this user is known or estimated. In MC CDMA the MMSE equaliser would require the knowledge of the channels of all active users or, as was done here, it requires the correlation matrix of the received signal to also be estimated.

\section{NUMERICAL RESULTS}

The numerical results presented were obtained for synchronous uplink MC CDMA and MC DS-CDMA systems employing BPSK modulation and orthogonal Hadamard sequences of length $M$. The $K$ channels are assumed identically distributed, and modelled as time-invariant FIR filters. The guard interval is of CP-type, and it is assumed its length $G_{I}$ is sufficiently large to allow IBI-free transmissions. For channel estimation purposes, each user transmits a burst of $N_{p}$ pilot symbols prior to the transmission of data symbols. The improved estimator assumes that the true CFR, which is to be estimated, lies in the subspace spanned by the first $G$ columns of $\mathbf{F}_{M, M}$.

First, an MC CDMA system with $M=32$ subcarriers is considered. This system serves $K=16$ active users. The system parameters of interest are $N_{p}=128$ and $G=8$.

Figure 3 depicts the NMSE of the conventional estimator $e_{c}^{2}$ in (27) and the lower and upper bounds on NMSE of the improved channel estimator $e_{w}^{2}$ in (35), versus the number of active users $K$.

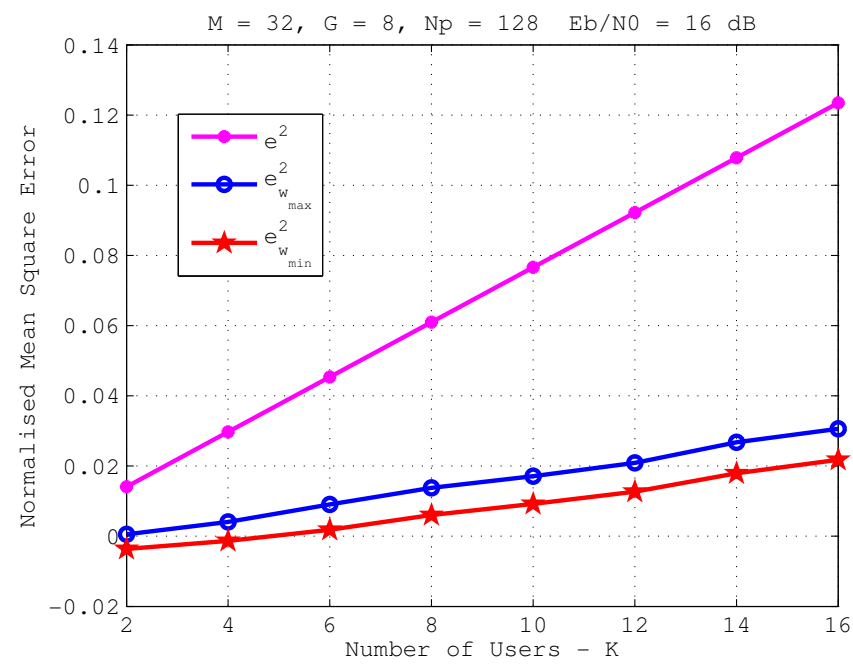

Fig. 3. Normalised Mean Square Error of conventional channel estimator $\left(e^{2}\right)$ versus number of active users $K$; Lower and Upper Bounds on Normalised Mean Square Error of improved channel estimator $\left(e_{w}^{2}\right)$ versus number of active users $K$, MC CDMA System

Since the general performance results depend on the particular choice of the signatures in use, the results presented here were obtained as follows: a group consisting of $K$ signatures drawn from the set of $M=32$ available spreading codes was assembled. Equations (27) and (35) were then computed for that group. Next, a new group consisting of $K$ signatures randomly selected was composed and the procedure was then repeated. The results presented thus correspond to the mean value of 100 random selections of groups of $K$ spreading sequences.

It is interesting to note that the lower and upper bounds on $e_{w}^{2}$ are indeed tight. It is also possible to verify that $e_{w}^{2}<e_{c}^{2}$, that is, the subspace projection estimator is indeed an improved estimator.

Figure 4 illustrates the lower and upper bounds on the MAI 
reduction factor $\eta$ in (36) as well as the noise reduction factor $\gamma=G / M$ in (34), versus the number of active users $K$. The results in Figure 4 were obtained by averaging $\eta_{\max }$ and $\eta_{\min }$ over 100 randomly selected groups containing $K$ signatures each. Since $\eta_{\max }$ in (36) is less than unity, $\eta$ indeed reduces the MAI component of the NMSE. Note that for loaded systems, since $\eta_{\max }<\gamma$, the reduction of the MAI component of the NMSE using the improved channel estimator is greater than the reduction of the noise component, which in turn does not depend on the system load. It is also worth mentioning that, for this example, the improved estimator reduces the noise component of the NMSE of the conventional estimator to $25 \%$ of its original value since $\gamma=\frac{G}{M}=0.25$. Moreover, it can be shown that when the system approaches full load $(K=32)$, the values of $\eta_{\min }$ and $\eta_{\max }$ converge to $\eta=\frac{G-1}{M-1}=0.226$, which is lower than $\gamma=\frac{G}{M}=0.25$, thus indicating that, on average, the reduction of the MAI component of the NMSE is more significant than the reduction of the noise component.

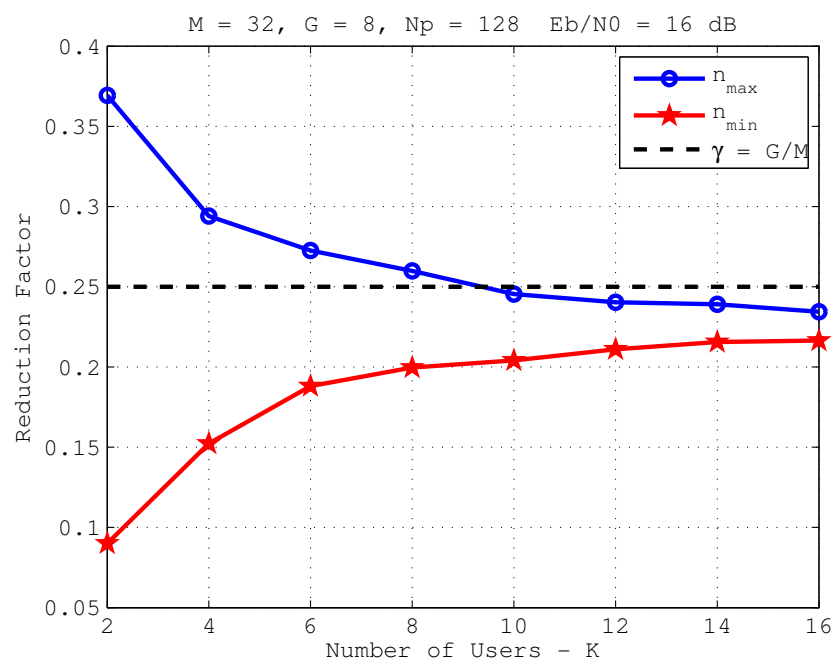

Fig. 4. Lower and Upper Bounds of the MAI Reduction Factor $\eta$ and noise reduction factor $\gamma$ versus number of active users $K$, MC CDMA system

The particular channel case described in section III-B is now considered. The time invariant coefficients are $h_{k_{i}}=p_{i} \alpha_{k_{i}}$, where $\alpha_{k_{i}}, i=0,1 \ldots, L-1$, are complex uncorrelated random variables with zero mean and $\mathrm{E}\left[\left|\alpha_{k_{i}}\right|^{2}\right]=1$. The weights $p_{i}$ satisfy $\sum_{i=0}^{L-1}\left|p_{i}\right|^{2}=1$. The channel scenario, referred herein as Channels Scenario A, considers uplink channels which have length $L=4$ with: $p_{0}=0.5957+j 0.0101, p_{1}=-0.3273-$ $j 0.3472, p_{2}=0.2910-j 0.0533, p_{3}=0.1285-j 0.5599$.

In Figure 5, the MAI reduction factor $\eta$ in (37) is evaluated for two MC CDMA systems with $M=16$ and $M=32$ subcarriers respectively, for Channels Scenario A. The other relevant parameter is $G=6$. The results shown are an average of 100 randomly selected groups composed of $K$ spreading sequences each.

Figure 6 assesses the average NMSE performance of the pilot-aided channel estimators obtained by means of Monte Carlo simulations of (1), in conjunction with (8) or (9), and compares them with numerical results dictated by expressions (27) and (38), for an MC CDMA system with $M=32$,

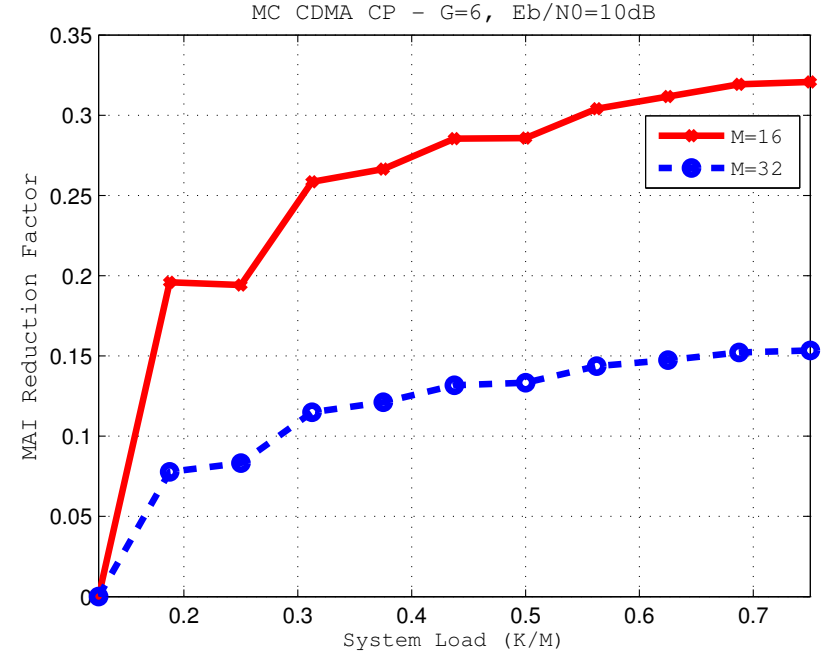

Fig. 5. MAI Reduction Factor $\eta$ versus system load $K / M$, MC CDMA system

$G=6, N_{p}=64$, experimenting Channels Scenario A. In all simulations the weights $\alpha_{k_{i}}$ are assumed as complex gaussian random variables. The values of $\alpha_{k_{i}}$ are randomly drawn and kept fixed throughout each simulation run. The simulation results are an average of 100 simulation runs. The results obtained by simulation matched the theoretical ones, and the improved channel estimate is less sensitive to increases in system loads.

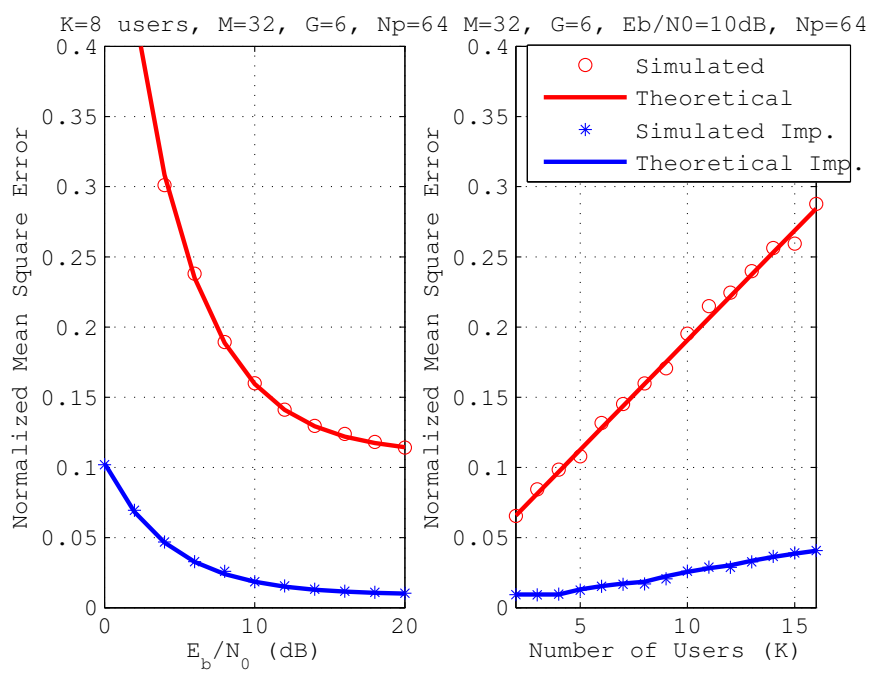

Fig. 6. NMSE performance of MC CDMA conventional and improved channel estimators, Channels Scenario A

A similar experiment is carried for a different channels scenario, referred as Channels Scenario B. Channels from Scenario $\mathrm{B}$ have the following weights: $p_{0}=0.74, p_{1}=-0.42$, $p_{2}=0.083, p_{3}=0.49, p_{4}=-0.12, p_{5}=0.01$ [34]. The MC CDMA system has the following parameters: $M=32, G=8$ and $N_{p}=128$. The simulated and theoretical NMSE of the conventional and improved estimators are depicted in Figure 7.

Figure 8 depicts NMSE performance of the channel esti- 

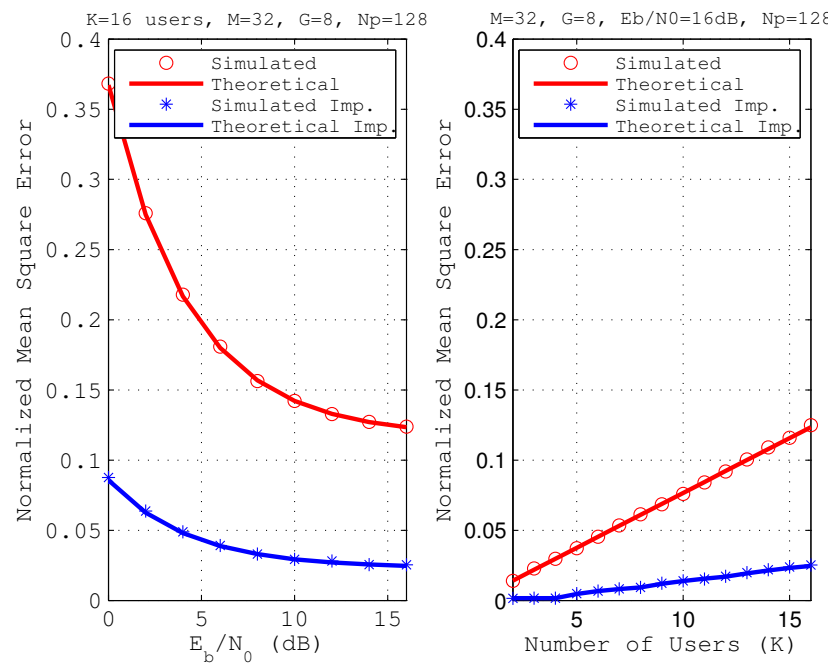

Fig. 7. NMSE performance of MC CDMA conventional and improved channel estimators, Channels Scenario B

mators for an MC DS-CDMA system with $M=32, G=6$, $N_{p}=64$ enduring Channels Scenario A. Results of Monte Carlo simulations of (39), in conjunction with (42) or (44), are compared with numerical results obtained from expressions (43) and (45). Since code orthogonality between users is preserved despite propagation through a multipath channel, the NMSE of the channel estimators are not affected by a MAI component.
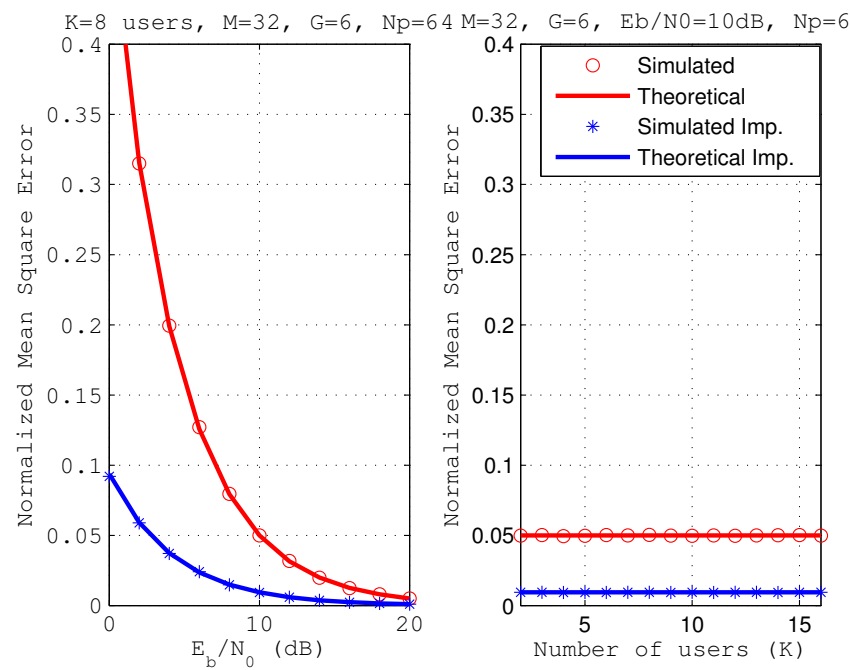

Fig. 8. NMSE performance of MC DS-CDMA conventional and improved channel estimators, Channels Scenario A

In order to evaluate the impact of the improved channel estimates on system performance, figures 9,10 and 11 present the average BER versus $E_{b} / N_{0}$ performance obtained from simulations.

Figure 9 depicts BER performance of an MC CDMA system with $M=32, G=8$ and $N_{p}=128$, for two different system loads, i.e. $K=8$ and $K=16$ active users, for Channels Scenario B. The results are an average of 500 Monte Carlo simulations of (1). For each simulation, a burst of $N_{p}$ pilot symbols is transmitted prior to transmission of up to $N_{t r}$ data symbols, where $N_{t r}$ is adequately selected for each $E_{b} / N_{0}$ considered. The CFR estimate obtained using the $N_{p}$ pilot symbols remains fixed during the reception of the $N_{t r}$ subsequent data symbols, over which BER is assessed. Symbol detection is carried by MMSE equalisation followed by hard decision, as described in Section III-C. The correlation matrix used in the MMSE equaliser is updated over the $N_{p}+N_{t r}$ transmitted symbols.

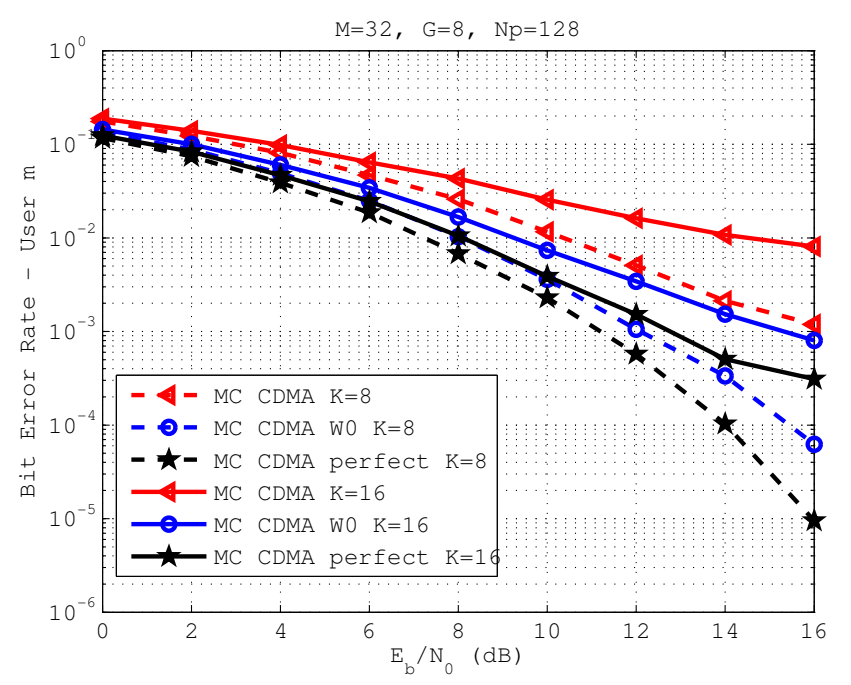

Fig. 9. BER versus $E_{b} / N_{0}$ performance for MC CDMA System, Channels Scenario B

Curves labeled "MC CDMA" refer to BER performance obtained using the MMSE equaliser of Section III-C in conjunction with the conventional CFR estimator, that is $\mathbf{w}_{m}=$ $\mathbf{R}^{-1} \mathbf{C}_{m} \widehat{\mathbf{q}}_{m}$ where $\widehat{\mathbf{q}}_{m}$ is the conventional estimate given by (8). Curves labeled "MC CDMA $W_{0}$ " refer to BER performance obtained using the MMSE equaliser in conjunction with the improved CFR estimator, that is $\mathbf{w}_{m}=\mathbf{R}^{-1} \mathbf{C}_{m} \widehat{\mathbf{q}}_{m}^{w}$ where $\widehat{\mathbf{q}}_{m}^{w}$ is the improved estimate given by (9). Curves labeled "MC CDMA perfect" refer to BER performance obtained using the MMSE equaliser in conjunction with the true CFR $\mathbf{q}_{m}$. Estimates of the signals' correlation matrix are obtained as $\widehat{\mathbf{R}}(i)=\frac{1}{i} \sum_{j=1}^{i} \mathbf{r}(j) \mathbf{r}^{H}(j)$. Since for large values of $E_{b} / N_{0}$ the correlation matrix becomes ill-conditioned, diagonal loading is carried in order to mitigate numerical instabilities. The results indicate that adoption of the simple channel estimator improving procedure provides a considerable gain in BER performance.

Figure 10 compares average BER versus $E_{b} / N_{0}$ performances of two MC CDMA systems, for Channels Scenario A: one with $M=32$ and the other with $M=16$; these two systems have the common parameters $K=16, G=6$ and $N_{p}=64$ or $N_{p}=128$. For $N_{p}=64$, the system with $M=32$ enjoys considerable gains in BER performance with the use of the channel improving procedure. Furthermore, for $N_{p}=128$, BER performance attained when using the improving procedure is close to the BER performance obtained for the case with perfect knowledge of the true CFR. Since the system with $M=16$ is fully loaded $(K=M)$ it 


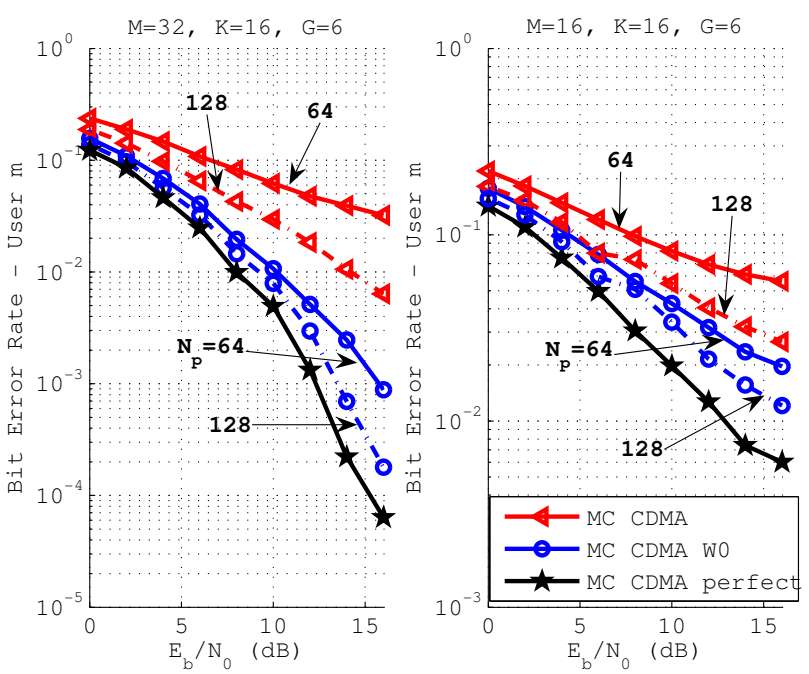

Fig. 10. BER versus $E_{b} / N_{0}$ performance for MC CDMA System, Channels Scenario A

performs poorly in terms of BER, as expected. Nevertheless, a receiver using the channel estimator improving procedure still offers gains of at least $4 \mathrm{~dB}$ over the BER performance of a receiver using the CFR conventional estimator. As expected, an increased number of transmitted pilot symbols yields better channel estimates which in turn translate into better BER performance.

It can be seen that when the improved estimators are used, the large reduction of the MAI component of the NMSE of the MC CDMA channel estimate translates into a significant improvement in performance.

Results of a similar experiment are illustrated in Figure 11, which depicts average BER versus $E_{b} / N_{0}$ performances of two MC DS-CDMA systems experimenting Channels Scenario A: one with $M=16, N_{p}=32$ and the other with $M=32$, $N_{p}=64$; these two systems share the common parameter $G=6$. The results are an average of 300 Monte Carlo simulations of (39). For each simulation, a burst of $N_{p}$ pilot symbols is transmitted prior to transmission of up to $N_{t r}$ data symbols, where $N_{t r}$ is adequately selected for each $E_{b} / N_{0}$ considered. The CFR estimate obtained using the $N_{p}$ pilot symbols remains fixed during the reception of the $N_{t r}$ subsequent data symbols, over which BER is assessed. Symbol detection is carried by MMSE equalisation followed by hard decision, as described in Section IV-B. It is assumed that the equaliser has perfect knowledge of the noise variance in (46). Curves labeled "MC DS-CDMA" refer to BER performance obtained using the MMSE equaliser of Section IV-B in conjunction with the conventional CFR estimator of (42) in (46). Curves labeled "MC DS-CDMA $W_{0}$ " refer to BER performance obtained using the MMSE equaliser in conjunction with the improved CFR estimator of (44) in (46). Curves labeled "MC DS-CDMA perfect" refer to BER performance obtained using the MMSE equaliser in conjunction with the true CFR $\mathbf{q}_{m}$. For both systems, there is approximately a $3 \mathrm{~dB}$ loss in performance when using the conventional CFR estimate in place of the perfect CFR. Nevertheless, for the system with $M=32$, the performance obtained with the adoption of the improved estimator is very close to the performance obtained when using perfect estimates. This is indeed expected, since adoption of the improving procedure reduces the variance of the noise induced estimation error by a factor of $\frac{G}{M}$, which evaluates to $\frac{6}{16}=0.375$ for $M=16$ and $\frac{6}{32}=0.1875$ for $M=32$.

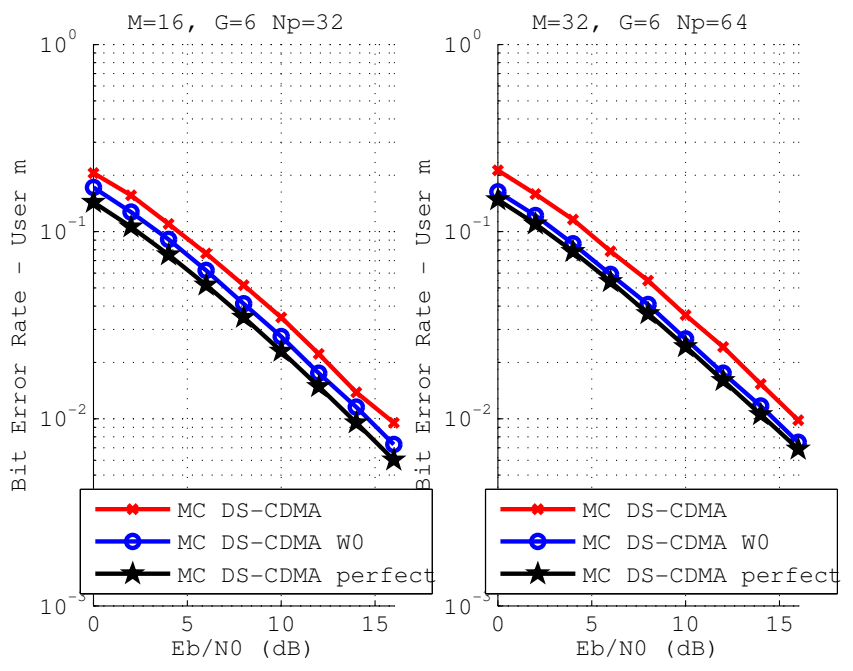

Fig. 11. BER versus $E_{b} / N_{0}$ performance for MC DS-CDMA System, Channels Scenario A

\section{Conclusions}

The problem of pilot-aided frequency-selective channel estimation for MC CDMA and MC DS-CDMA has been addressed. Performance in terms of NMSE were derived for two channel estimators: a conventional estimator based on the minimum mean square error criterion, and an improved estimator which exploits subspace relationships between the frequency and impulse responses of the discrete channel.

For MC DS-CDMA, NMSE performance of both estimators resulted, thanks to MAI suppression, in simple closed-form solutions which depend only on the number of carriers $M$, number of pilot symbols $N_{p}$ and noise power $\sigma^{2}$, for the conventional estimator, and dimension $G$ of the subspace in which the true CFR is assumed to lie, number of pilot symbols $N_{p}$ and noise power $\sigma^{2}$, for the improved estimator.

Upper and lower bounds on NMSE were derived for both estimators in the case of MC CDMA systems. Upper and lower bounds of the conventional estimator degenerate into the same expression, thus resulting in a closed-form NMSE, which depends only on the number of pilot symbols $N_{p}$, number of active users $K$, number of carriers $M$ and noise power $\sigma^{2}$. Furthermore, assuming the $K$ channels $i$ ) share a common power delay profile and $i$ ) are identically distributed, results in a closed-form expression for the NMSE of the improved estimator, which in turn depends only on the number of pilot symbols $N_{p}$, dimension $G$ of the subspace in which the true CFR is assumed to lie, noise power $\sigma^{2}$ as well as the common power delay profile and spreading sequences in use.

Observation of the obtained NMSE expressions indicated that MAI and thermal noise contribute differently to the 
performance of the estimators. The fact that the improving procedure via projection matrix affects differently the thermal noise and MAI terms of the NMSE of the improved estimator motivated the definitions of a noise reduction factor as well as a MAI reduction factor.

\section{APPENDIX}

This Appendix shows the reduction in estimation error resulting from the use of projection matrix $\mathbf{W}_{o}$. Let $\widehat{\mathbf{q}}$ be an estimate of the $M \times 1$ vector $\mathbf{q}, \widehat{\mathbf{q}}=\mathbf{q}+(\widehat{\mathbf{q}}-\mathbf{q})=\mathbf{q}+\varepsilon$, where $\varepsilon$ is the error vector incurred by the estimation procedure.

Suppose vector $\mathbf{q}$ is of the form $\mathbf{q}=\mathbf{F h}$, i.e, $\mathbf{q}$ lies in the subspace spanned by the columns of matrix $\mathbf{F}$ (with $\mathbf{h}$ of dimension $L \times 1$, and $L \leq M)$. Let $\mathbf{W}_{o}$ be the matrix that projects a vector $\widehat{\mathbf{a}} \in \mathrm{C}^{M}$ in this subspace:

$$
\mathbf{W}_{0}=\mathbf{F}\left(\mathbf{F}^{H} \mathbf{F}\right)^{-1} \mathbf{F}^{H} \text {. }
$$

Thus the projection $\widehat{\mathbf{q}}_{p}$ of $\widehat{\mathbf{q}}$ in the subspace spanned by the columns of $\mathbf{F}$ is given by:

$$
\widehat{\mathbf{q}}_{p}=\mathbf{W}_{0} \widehat{\mathbf{q}}=\mathbf{W}_{0} \mathbf{q}+\mathbf{W}_{0} \varepsilon=\mathbf{q}+\varepsilon_{p},
$$

where $\varepsilon_{p}$ is the projection of the error vector $\varepsilon$ in the subspace spanned by $\mathbf{F}$. Since $\varepsilon \in \mathrm{C}^{M}$,

$$
\varepsilon=\mathbf{W}_{o}^{\perp} \varepsilon+\mathbf{W}_{o} \varepsilon=\mathbf{W}_{o}^{\perp} \varepsilon+\varepsilon_{p},
$$

where $\mathbf{W}_{o}^{\perp}=\left(\mathbf{I}-\mathbf{W}_{o}\right)$. Thus,

$$
\|\varepsilon\|^{2}=\left\|\mathbf{W}_{o}^{\perp} \varepsilon\right\|^{2}+\left\|\varepsilon_{p}\right\|^{2}+2 \operatorname{Re}\left[\varepsilon^{H} \mathbf{W}_{o}^{H} \mathbf{W}_{o}^{\perp} \varepsilon\right] .
$$

Since $\mathbf{W}_{o}$ is a projection matrix, $\mathbf{W}_{o}^{H}=\mathbf{W}_{o}$ and $\mathbf{W}_{o}^{2}=\mathbf{W}_{o}$ hold, resulting that $\mathbf{W}_{o} \mathbf{W}_{o}^{\perp}=0$. It then follows from (50) that

$$
\left\|\varepsilon_{p}\right\|^{2} \leq\|\varepsilon\|^{2}
$$

\section{REFERENCES}

[1] K. S. Guilhousen, I. M. Jacobs, R. Padovani, A. J. Viterbi, A. Weaver C. E. Wheatley, "On the Capacity of a Celular CDMA System," IEEE Trans. Veh. Technol., vol. 40, pp. 303-311, May 1991.

[2] A. J. Viterbi, Principles of Spread Spectrum Communications. AddisonWesley, 1995.

[3] K. Tachikawa, W-CDMA Mobile Communications Systems. John Wiley \& Sons Inc., 2002.

[4] E. Dahlman, B. Gudmundson, M. Nilsson, J. Skold, "UMTS/IMT-2000 based on Wideband CDMA," IEEE Commun. Mag., vol. 36, no. 9, pp. 70-80, Sep. 1998.

[5] R. W. Chang, "Synthesis of band-limited orthogonal signals for multichannel data transmission," Bell Systems Technical Journal, no. 46, pp. 1775-1796, 1966.

[6] R. W. Chang, R. A. Gibbey, "A theoretical study of performance of an orthogonal multiplexing data transmission scheme," IEEE Trans. Commun. Technol., vol. 16, no. 4, pp. 529-540, 1968.

[7] B. R. Saltzberg, "Performance of an efficient parallel data transmission system," IEEE Trans. Commun. Technol., vol. 15, no. 6, pp. 805-811, 1967.

[8] S. B. Weinstein, P. M, Ebert, "Data transmission by frequency division multiplexing using the discret Fourier transform," IEEE Trans. Commun. Technol., COM-19(5), pp. 628-634, Oct. 1971.

[9] S. Kondo, L. B. Milstein, "On the use of multicarrier direct sequence spread spectrum systems," in Proc. IEEE Military Communications Conf. (MILCOM), 1993, pp. 52-56.

[10] V. DaSilva, E. S. Sousa, "Performance of Orthogonal CDMA Codes for Quasi-synchronous Communication Systems," in Proc. IEEE Int. Conf. Universal Personal Communications (ICUPC), 1993, pp.995-999.

[11] Z. Wang, G. B. Giannakis, "Wireless Multicarrier Communications," IEEE Signal Process. Mag., pp. 29-48, May 2000.
[12] F. Petre, G. Leus, M. Moonen, H. De Man, "Multicarrier block-spread CDMA for broadband cellular downlink," EURASIP Journal on Applied Signal Processing, no. 10, pp. 1568-1584, 2004.

[13] G. B. Giannakis, P. A. Anghel, Z. Wang, "Generalized multicarrier CDMA: unifcation and linear equalization," EURASIP Journal on Applied Signal Processing, no. 5, pp. 743-756, 2005.

[14] B. Muquet, Z. Wang, G. B. Giannakis, M. de Courville, P. Duhamel, "Cyclic Prefixing or Zero Padding for Wireless Multicarrier Transmissions?," IEEE Trans. Commun., vol. 12, no. 50, pp. 2136-2148, 2002.

[15] N. Yee, J. P. Linnartz, "Wiener Filtering of Multi-Carrier CDMA in Rayleigh Fading Channel," in Proc. IEEE Symp. Personal Indoor and Mobile Radio Communications (PIMRC'94), 1994, vol. 4, pp. 13441347.

[16] H. Xing, M. Renfors, "The performance evaluation of the Multicarrier CDMA system with frequency domain equalization," in Proc. IEEE Vehicular Technology Conf. (VTC'99), 1999, vol. 4, pp. 2362-2366.

[17] S. Kaiser, P. Hoecher, "Performance of multicarrier CDMA with channel estimation in two dimensions," in Proc. IEEE Symp. Personal Indoor and Mobile Radio Communications (PIMRC'97), 1997, vol. 1, pp. 115-119.

[18] P. Hoeher, S. Kaiser, P. Robertson, "Two-dimensional pilot-symbolaided channel estimation by Wiener filtering," in Proc. IEEE Int. Conf. Acoustics, Speech and Signal Processing (ICASSP'97), 1997, vol. 3, pp. 1845-1848.

[19] A. Nagate, T. Fujii, "A study on channel estimation methods for time-domain spreading MC-CDMA systems," IEEE Trans. Wireless Commun., vol. 7, pp. 5233-5237, Dec. 2008.

[20] B. S. Chen, J. F. Liao, "Adaptive MC-CDMA multiple channel estimation and tracking over time-varying multiple fadding channels," IEEE Trans. Wireless Commun., vol. 6, no. 6, pp. 2328-2337, Jun. 2007.

[21] D. N. Kalofonos, M. Stojanovic, J. G. Proakis, "Performance of Adaptative MC-CDMA detectors in rapidly fading Rayleigh channel," IEEE Trans. Wireless Commun., vol. 2, no. 2, pp. 1375-1387, Mar. 2003.

[22] S. Kondo, B. Milstein, "Performance of multicarrier DS-CDMA systems," IEEE Trans. Commun., vol. 44, pp. 238-246, Feb. 1996.

[23] J. Wang, H. Huang, "MC DS/SFH-CDMA systems for overlay systems," IEEE Trans. Wireless Commun., vol. 1, no. 3, pp. 448-455, Jul. 2002.

[24] P. Marques, A. Gameiro, J. Fernandes, "Vectorial channel estimation for uplink MC-CDMA in beyond 3G wireless systems," in Proc. IEEE Symp. Personal Indoor and Mobile Radio Communications (PIRMC'03), 2003.

[25] L. Tong, B. M. Sadler, M. Dong, "Pilot-assisted wireless transmissions: general model, design criteria and signal processing," IEEE Signal Process. Mag., vol. 21, pp. 12-25, Nov. 2004.

[26] R. B. Moraes, "Dynamic Allocation of Pilot-Symbols in Closed-Loop OFDM Systems," M.S. thesis, Dept of Electrical Engineering, PUC-Rio, Rio de Janeiro, Brazil, 2009. (in Portuguese)

[27] X. G. Doukopoulos, G. V. Moustakides, "Blind adaptive channel estimation in OFDM systems," IEEE Trans. Wireless Commun., vol. 5, no. 7, pp. 1716-1725, Jul. 2006.

[28] J. Van de Beek. O. Edfors, M. Sandell, S. Wilson, P. Brjesson, "On channel estimation for OFDM systems," Proc. of the Vehicular Technology Conf., Chicago, vol. 2, pp. 815-819, Jul. 1995.

[29] D. F. Cardoso, F. D. Backx, R. Sampaio-Neto, "Performance of Multicarrier CDMA Systems with Improved Pilot-Aided Channel Estimation," in Proc. IEEE 6th Int. Conf. on Wireless On-demand Network Systems and Services (WONS2009), Snowbird, USA, 2009.

[30] D. F. Cardoso, F. D. Backx, R. Sampaio-Neto, "Improved Pilot-Aided Channel Estimation in Zero Padded MC-CDMA Systems," in Proc. IEEE 4th Int. Symp. on Wireless Pervasive Computing (ISWPC2009), Melbourne, Australia, 2009.

[31] L. Sanguinetti, M. Morelli, "Multiuser Channel Estimation and Tracking for MC-CDMA Uplink Transmissions," Proc. IEEE 16th Int. Symp. on Personal, Indoor and Mobile Radio Communications (PIMRC2005), 2005, vol. 1, pp. 251-256.

[32] Ye G. Li, G. L. Stuber, Orthogonal Frequency Division Multiplexing for Wireless Communications. Springer, 1st ed, 2006.

[33] A. Scherb, V. Kuehn, K.-D. Kammeyer, "Pilot aided channel estimation for short code DS-CDMA", in Proc. IEEE 7th Int. Symp. on Spread Spectrum Techniques and Applications (ISSSTA2002), 2002, pp. 39-43.

[34] H. Sari, G. Karam, I. Jeanclaude, "Transmission techniques for Digital Terrestrial TV Broadcasting," IEEE Commun. Mag., pp. 100-109, 1995. 


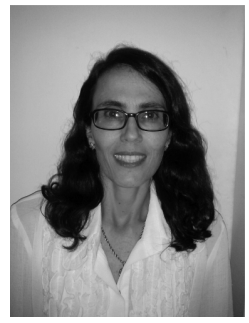

Deolinda Fontes Cardoso received the Diploma degree in Electrical Engineering from Pontifícia Universidade Católica do Rio de Janeiro (PUC-Rio), Brazil, in 1987, the M.Sc. degree in Computer Science from Universidade Federal Fluminense, Brazil, in 2004, and the D.Sc. degree in Electrical Engineering from PUC-Rio, in 2008. Since 1987, she has been an Electrical Engineer in the Brazilian Navy.

Her research interests include multicarrier systems, spread spectrum, parallel processing in cloud computing and software security assurance.

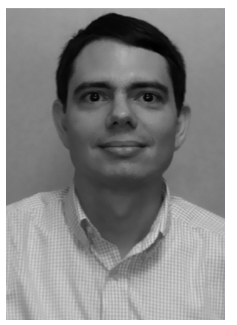

Fabian David Backx received the Diploma degree in Telecommunications Engineering from Universidade Federal Fluminense, Brazil, in 2002, and the M.Sc. and D.Sc. degrees, both in Electrical Engineering, from Pontifícia Universidade Católica do Rio de Janeiro, Brazil, in 2004 and 2009, respectively. Since 2010, he has been a researcher at Instituto de Pesquisas da Marinha, Brazil.

His research interests are statistical signal processing, communication theory, digital transmission and signal processing for communications and radar.

$\mathrm{He}$ is a member of the IEEE and of the Brazilian Telecommunications Society (SBrT).

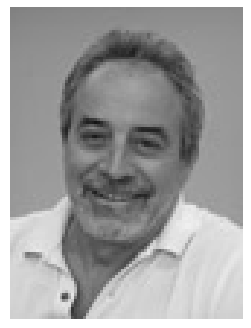

Raimundo Sampaio-Neto received the Diploma and the M.Sc. degrees, both in Electrical Engineering, from Pontifícia Universidade Católica do Rio de Janeiro (PUC-Rio), Brazil, in 1975 and 1978, respectively, and the Ph.D. degree in Electrical Engineering from the University of Southern California (USC), Los Angeles, USA, in 1983.

From November 1983 to June 1984 he was a Post-Doctoral fellow at the Communication Sciences Institute of the Department of Electrical Engineering at USC, and a member of the technical staff of Axiomatic Corporation, Los Angeles. He is now a researcher at the Center for Studies in Telecommunications (CETUC) and an Associate Professor of the Department of Electrical Engineering of PUC-Rio, where he has been since July 1984. During 1991 he was a Visiting Professor in the Department of Electrical Engineering at USC. Prof. Sampaio-Neto has participated in various projects and consulted for several private companies and government agencies.

$\mathrm{He}$ was co-organizer of the Session on Recent Results for the IEEE Workshop on Information Theory, 1992, Salvador, Brazil. He has also served as Technical Program co-Chairman for IEEE Global Telecommunications Conference (Globecom'99) held in Rio de Janeiro in December 1999 and as a member of the technical program committees of several national and international conferences. He was in office for three terms for the Board of Directors of the Brazilian Communications Society. He served as a member of its Advisory Council for four terms and as Associate Editor of the society journal, Journal of Communication and Information Systems.

His areas of interest include satellite communications, communication theory, digital transmission and signal processing for communications, areas in which he has published more than 160 papers in referred journals and conferences. 